\title{
Optical Fiber Sensors for Rapid Screening of COVID-19
}

\author{
Pooja $\mathrm{Nag}^{1,2} \cdot$ Kapil Sadani ${ }^{1,3} \cdot$ Soumyo Mukherji ${ }^{1}[$
}

Received: 16 April 2020 / Revised: 3 June 2020 / Accepted: 11 June 2020 / Published online: 19 June 2020

(C) Indian National Academy of Engineering 2020

\begin{abstract}
Rapid diagnosis of coronavirus disease COVID-19 is challenging in developing countries due to diverse clinical presentations and limited healthcare infrastructure. Biosensors hold immense prospects for diagnosis of the disease. Two approaches are proposed: the first involves measurement of host immune response and second, the detection of viruses or viral cell surface proteins using suitable bioreceptors. The article provides an overview of evanescent wave absorbance and localized surface plasmon resonance-based optic fiber platform for potential screening of COVID-19.
\end{abstract}

Keywords COVID-19 SARS-CoV-2 $\cdot$ Evanescent wave absorbance $\cdot$ Localized surface plasmon resonance $\cdot$ Optical fiber sensor

\section{Introduction}

The coronavirus disease (COVID-19) caused by severe acute respiratory syndrome coronavirus 2 (SARS-CoV-2) is marked by one or more of symptoms: high fever, dyspnea, diarrhea, throat ache, headache, fatigue, loss of sense of taste and smell (Menni et al. 2020). The diversity of clinical presentations of this disease has made it challenging for rapid clinical diagnosis. Reverse transcriptase RT-PCR coupled quantitative PCR is the only established gold standard method for definitive detection of COVID-19 by nucleic acid sequencing. Therapeutics for most viral infections, in general, are vaccines administered to trigger an immune response and thereafter remain as an immunological memory in the host immune system.

Two alternative approaches for viral infection diagnostics are possible and practiced: the first involves serological

Pooja Nag and Kapil Sadani have contributed equally.

Soumyo Mukherji

mukherji@iitb.ac.in

1 Department of Biosciences and Bioengineering, Indian Institute of Technology Bombay, Mumbai 400076, India

2 Department of Mechatronics Engineering, Manipal Institute of Technology, Manipal Academy of Higher Education, Manipal 576104, India

3 Department of Instrumentation and Control, Manipal Institute of Technology, Manipal Academy of Higher Education, Manipal 576104, India investigations for measurement of elevated biomarker levels, for example, measurement of immunoglobin $\mathrm{M}(\operatorname{IgM})$ and immunoglobin $\mathrm{G}(\mathrm{IgG})$, while the second involves direct determination of the virus itself, utilizing its unique cellular proteins. The former approach of immunosensing is limited by the concentration and half-life of the marker itself (generally host antibodies). But, in the case of acute diseases such as COVID-19, these may serve as useful pre-screening tests. The second approach involves direct detection of viruses by its cell surface proteins. For detection of newer viruses such as SARS-CoV-2, identification of unique antigen is necessary. A preliminary investigation reveals that the SARS-CoV-2 has four major structural proteins, namely the $\mathrm{S}$ (spike), E (envelope), M (membrane), and N (nuleocapsid) proteins (Fig. 1). Previous studies on coronaviruses including that of SARS-CoV and MERS-CoV mostly report utilization of the S1 RBD (subunit 1 receptor binding domain of $\mathrm{S}$ protein) and $\mathrm{N}$ proteins as antigens to develop detection assays. Commonly used receptors for these are antibodies (Li et al. 2020), nanobodies (Zhou et al. 2018), or aptamers (Ahn et al. 2009).

Biosensors hold immense prospects in reliable and affordable diagnostic development, particularly in developing countries working with limited infrastructure, resources, and skills. The development of biosensors involves integration of three key components: the receptor, analyte-receptor interaction interface, and transduction platform. Direct determination of SARS-CoV-2 should be possible using specific receptors to the S1 RBD antigen, while detection of host 


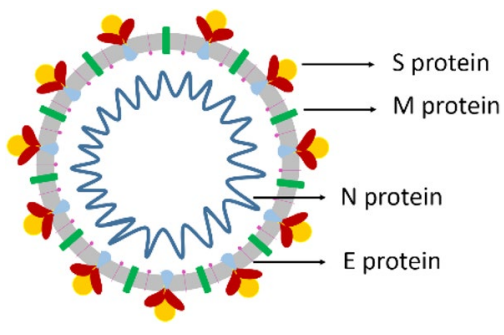

Fig. 1 Structural proteins of SARS-CoV-2

immune response would involve serological investigation of IgG and IgM. Receptor-analyte interactions are generally recorded on one of optical, electrochemical, mass, acoustic or hybrid transduction platforms as an electronic readout. Our group specializes in development of optic fiber sensors which offer several advantages such as immunity to electrostatic and electromagnetic interferences, robustness, portability, and ultrasensitive detection limits for direct, label-free detection of analyte. Customized interfaces such as plasmonic gold nanoparticles, silver nanoparticles, their hybrids, and electroactive conducting polymers such as polyaniline and polypyrrole nanofibers have been utilized as effective interfaces for both immunosensing and specific analyte detection. This report explores the possible approaches of development of a point of care, low-cost evanescent wave absorbance (EWA)-based optical fiber sensor for quick and specific diagnosis of SARS-CoV-2.

\section{EWA-Based Optical Fiber Sensor Technology}

Light is propagated through an optical fiber through total internal reflection. At each such reflections at the interface, a part of the energy of the guided wave is lost to the cladding and forms an electromagnetic field called the evanescent wave. The wave is very sensitive to changes in refractive index at the interface and this property is utilized to develop EWA-based fiber optic sensors. A certain portion of the fiber is decladded to expose the core so that the evanescent wave penetrates into the surrounding medium. EWA-based optic fiber sensing systems involve use of different tapered and curved geometries of the sensing region to achieve maximal penetration depth for transduction of receptor-analyte interactions at the core-clad interface (Punjabi et al. 2015). A schematic of the optic fiber sensing setup is illustrated in Fig. 2a. Light is coupled into the optical fibers from a light emitting diode (LED) and measured at the output by a suitable photodetector. Analyte-receptor interaction in the decladded (usually U-bend) region effects a localized refractive index change which is measured as a change in absorbance. This principle has been also utilized to develop highly sensitive localized surface plasmon resonance (LSPR)-based sensor, where, metal nanoparticles are immobilized on the sensor surface. Additionally, in novel interfaces such as polyaniline nanofibers, such interactions effect a localized change in charge distribution of the interface which is also utilized for biosensing.

The EWA and LSPR technologies have been utilized for varied applications, such as, immune sensing, detection of bacteria, heavy metals (Sadani et al. 2019), protein biomarkers (Khatri et al. 2018), antibiotics, and bacterial susceptibility (Nag et al. 2020). Such sensors have been able to detect bacteria upto $60 \mathrm{CFU} / \mathrm{ml}$ (polyaniline-coated optical fiberbased EWA sensor) and less than $1000 \mathrm{CFU} / \mathrm{ml}$ (in EWA sensor) bacteria in water matrices (Bharadwaj et al. 2011). Further, this has been also translated to a point of use plug and play system as demonstrated in Fig. 2b (Chandra et al. 2018). The novelty of each work lies in the optimization of receptors for detection of the analyte.

\section{Proposed Scheme for Diagnosis of SARS-CoV-2}

In response to an infection, the body initially produces $\operatorname{IgM}$. This is subsequently replaced by IgG-type antibodies, which can be detected in blood days after the infections have been (a)

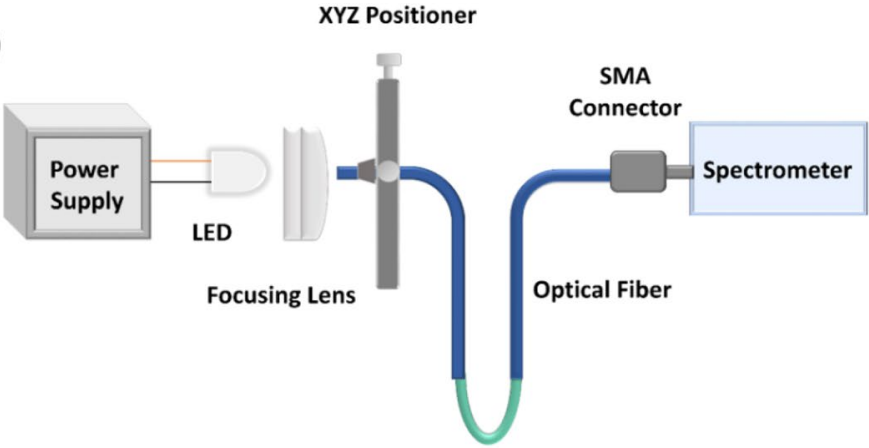

(b)

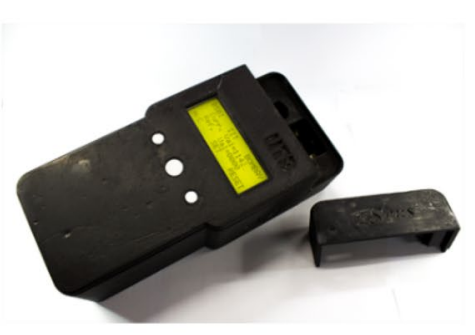

Fig. 2 a Optical detection assembly. b Hand-held device developed for point of care use (reused with permission) 
Fig. 3 Schematic representations of the two proposed sensing schemes. a Direct detection of SARS-CoV-2 with immobilized antibodies. b Detection of IgG or IgM with immobilized specific proteins on gold nanoparticles/polyaniline nanofiberscoated optical fiber (a)

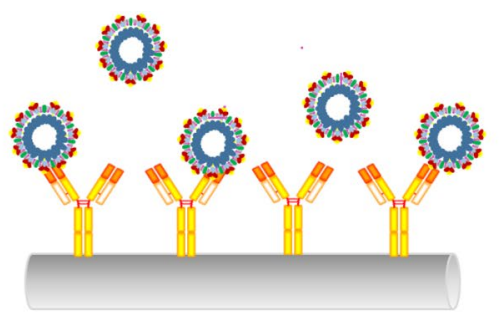

(b)

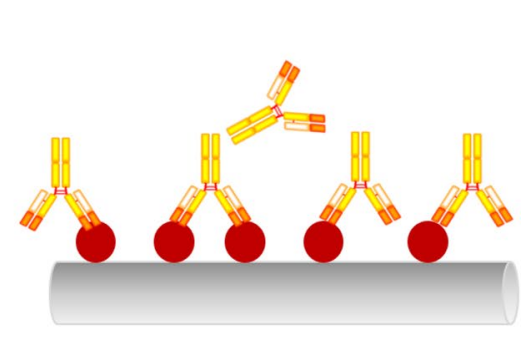

SARS-CoV-2
Antigen
Antibody

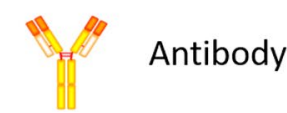

cleared. Thus, IgM and IgG levels in blood/ serum can be used as an indicator for exposure to SARS-CoV-2. Lateral flow assay-based tests, such as the one developed by Abbott are based on this very principle. If suitable receptors be available, biosensor cartridges can be indigenously developed and upscaled for such serological measurements in a plug and play format. Given the high sensitivity of EWA- and LSPR-based technologies, such sensors should be able to detect low concentrations of the immunoglobins, thereby reducing chances of false negatives and detection at an early stage. In an alternate approach, the antibodies for the unique $\mathrm{S} 1$ subunit of the $\mathrm{S}$ protein can be used to detect the virus itself in swab samples down to an LoD of at least 100 units/ml within an hour.

Figure 3 shows the two proposed schemes for development of such sensors. Figure 3a shows antibodies immobilized on either gold nanoparticle or polyaniline-coated optical fibers for specific sensing of the virus. While Fig. $3 b$ has specific surface proteins (of the virus) immobilized on the optical fiber for detection of IgG and/or IgM (produced as an immune response). In Scheme 3(a), the binding of virus to the antibodies would effect a localized change in the refractive index causing a change in the light intensity (absorbance) at the output. In the case of conducting polymers, this change in dielectric would cause a change in localized charge distribution, causing a change in the absorbance at the output. In the second scheme, the antigen is non-specifically captured on the optical fiber using a homo-bi-functional linker such as glutaraldehyde, or by carbodiimide-ester chemistries. Recognition of the pathogenic protein using appropriate antibodies would cause a localized refractive index change to the same effect as in the earlier case. The change in the output signal would typically be dependent on the concentration of analyte present in the sample.

\section{Conclusion}

There is tremendous scope and potential in biosensor development for detection of COVID-19. It would, however, be inappropriate to compare these with the RT-PCR-coupled
qPCR methods in terms of diagnostic accuracy for two primary concerns: first, host immune responses are not precise indicators of current infection or progression of disease and second, other respiratory pathogens such as SARS-CoV or MERS might trigger similar immune responses. Direct determination of SARS-CoV-2 by its unique cell surface proteins is likely to provide more accurate results with a possible insight into the localized viral load. Biosensors could serve as useful pre-screening tests of COVID-19, particularly in countries like India. With a proven capacity of immunosensing down detection limits of $37 \mathrm{pM}$ (Chandra et al. 2017) and various specific analyte detections, our optic fiber platform holds tremendous prospects in COVID19 screening.

\section{References}

Ahn DG, Jeon IJ, Kim JD, Han SR, Lee SW, Jung H, Oh JW (2009) RNA aptamer-based sensitive detection of SARS coronavirus nucleocapsid protein. Analyst 134:1896-1901. https://doi. org/10.1039/b906788d

Bharadwaj R, Sai VVR, Thakare K, Dhawangale A, Kundu T, Titus S, Verma PK, Mukherji S (2011) Evanescent wave absorbance based fiber optic biosensor for label-free detection of E. coli at $280 \mathrm{~nm}$ wavelength. Biosens Bioelectrons 26:3367-3370

Chandra S, Bharadwaj R, Mukherji S (2017) Label free ultrasensitive optical sensor decorated with polyaniline nanofibers: Characterization and immunosensing application. Sens Actuators B Chem 240:443-450. https://doi.org/10.1016/j.snb.2016.08.103

Chandra S, Dhawangale A, Mukherji S (2018) Hand-held optical sensor using denatured antibody coated electro-active polymer for ultra-trace detection of copper in blood serum and environmental samples. Biosens Bioelectrons 110:38-43

Khatri A, Punjabi N, Ghosh D, Maji SK, Mukherji S (2018) Detection and differentiation of $\alpha$-synuclein monomer and fibril by chitosan film coated nanogold array on optical sensor platform. Sens Actuators B Chem 255:692-700. https://doi.org/10.1016/j. snb.2017.08.051

Li Z, Yi Y, Luo X, Xiong N, Liu Y, Li S, Sun R, Wang Y, Hu B, Chen W, Zhang Y, Wang J, Huang B, Lin Y, Yang J, Cai W, Wang X, Cheng Z, Sun K, Pan W, Zhan Z, Chen L, Ye F (2020) Development and clinical application of a rapid IgM-IgG combined antibody test for SARS-CoV-2 infection diagnosis. J Med Virol. https://doi.org/10.1002/jmv.25727 
Menni C, Valdes AM, Freidin MB et al (2020) Real-time tracking of self-reported symptoms to predict potential COVID-19. Nat Med. https://doi.org/10.1038/s41591-020-0916-2

Nag P, Sadani K, Mukherji S, Mukherji S (2020) Beta-lactam antibiotics induced bacteriolysis on LSPR sensors for assessment of antimicrobial resistance and quantification of antibiotics. Sens Actuators B Chem 311:127945. https://doi.org/10.1016/j. snb.2020.127945

Punjabi N, Satija J, Mukherji S (2015) Evanescent wave absorption based fiber-optic sensor-cascading of bend and tapered geometry for enhanced sensitivity, sensing technology: current status and future trends III. Smart Sens Meas Instrum. https://doi. org/10.1007/978-3-319-10948-0_2

Sadani K, Nag P, Mukherji S (2019) LSPR based optical fiber sensor with chitosan capped gold nanoparticles on BSA for trace detection of $\mathrm{Hg}(\mathrm{II})$ in water, soil and food samples. Biosens Bioelectron 134:90-96. https://doi.org/10.1016/j.bios.2019.03.046

Zhou G, He L, Sun S, Qiu H, Tai W, Chen J, Li J, Chen Y, Guo Y, Wang Y, Shang J, Ji K, Fan R, Du E, Jiang S, Li F, Du L, Zhou Y, Virol J (2018) A novel nanobody targeting middle east respiratory syndrome coronavirus (MERS-CoV) receptor-binding domain has potent cross-neutralizing activity and protective efficacy against MERS-CoV. Analyst 92:e00837-e918. https://doi.org/10.1039/ b906788d

Publisher's Note Springer Nature remains neutral with regard to jurisdictional claims in published maps and institutional affiliations. 